\title{
Motion-in-depth effects on interceptive timing errors in an immersive enviornment
}

Joan López-Moliner ( $\boldsymbol{\sim}$ j.lopezmoliner@ub.edu )

Universitat de Barcelona

Cristina Malla

Universitat de Barcelona

\section{Research Article}

Keywords: shooting, interception, fronto-parallel plane, hereafter TTC, Brouwer, Smeets, Brenner

Posted Date: July 26th, 2021

DOl: https://doi.org/10.21203/rs.3.rs-738211/v1

License: (c) (i) This work is licensed under a Creative Commons Attribution 4.0 International License. Read Full License 


\title{
Motion-in-depth effects on interceptive timing errors in an immersive environment
}

\author{
Joan López-Moliner ${ }^{1},{ }^{*}$ and Cristina de la Malla ${ }^{1}$ \\ ${ }^{1}$ Vision and Control of Action (VISCA) Group, Department of Cognition, Development and \\ Psychology of Education, Institut de Neurociències, Universitat de Barcelona, Barcelona, \\ Catalonia, Spain \\ *Corresponding author: j.lopezmoliner@ub.edu
}

\begin{abstract}
We often need to interact with targets that move along arbitrary trajectories in the 3D scene. In these situations, information of parameters like speed, time-to-contact, or motion direction is required to solve a broad class of timing tasks (e.g., shooting, or interception). There is a large body of literature addressing how we estimate different parameters when objects move both in the fronto-parallel plane and in depth. However, we do not know to which extent the timing of interceptive actions is affected when there is MID involved. Unlike previous studies that have looked at the timing of interceptive actions using constant distances and fronto-parallel motion, we here use immersive virtual reality to look at how differences in the above-mentioned variables influence timing errors in a shooting task performed in a 3D environment. Participants had to shoot at targets that moved following different angles of approach with respect to the observer when those reached designated shooting locations. We recorded the shooting time, the temporal and spatial errors and the head's position and orientation in two conditions that differed in the interval between the shot and the interception of the target's path. Results show a consistent change of the temporal error across approaching angles: the larger the angle, the earlier the error. Interestingly, we also found different error patterns within a given angle that depended on whether participants tracked the whole target's trajectory or only its end-point. These differences had larger impact when the target moved
\end{abstract}


in depth and are consistent with underestimating motion-in-depth in the periphery. We conclude that the strategy participants use to track the trajectory interacts with MID and affects timing performance.

\section{Introduction}

In daily-life we often need timing our actions with moving objects that can travel at different speeds and in different directions (e.g., in the fronto-parallel plane or in depth). In order to succeed in tasks that require the timing of actions with moving targets at particular times or locations, people would certainly benefit from ascertaining parameters like the target's speed, time-to-contact (hereafter TTC) or motion direction. Knowledge of such parameters can help people to better predict future positions of the object. Moreover, information such as velocity can be used to update targets' changing positions (Kwon, Tadin, \& Knill, 2015) even in timing tasks (Aguilar-Lleyda, Tubau, \& López-Moliner, 2018). Despite factors such as the distance of the object, its trajectory and the trajectory with respect to the observer potentially influencing the retinal speed of the object, speed estimates should remain approximately constant to properly interact with moving targets.

The use of visual fronto-parallel motion has predominated in studies aiming at understanding how we time our movements, either making targets move with linear motion (e.g. Aguilar-Lleyda et al., 2018; Brenner \& Smeets, 2009; Brouwer, Smeets, \& Brenner, 2005; Cámara, López-Moliner, Brenner, \& Malla, 2020; de la Malla \& López-Moliner, 2015a; de la Malla, López-Moliner, \& Brenner, 2012; de la Malla, Smeets, \& Brenner, 2018) or following parabolic flights on the frontal plane (Joerges \& LópezMoliner, 2019; Kreyenmeier, Fooken, \& Spering, 2017). Thus, despite the relevance of motion-in-depth (MID) for daily life interceptive timing (think of a football goalkeeper, or a baseball batter) its effects have been mostly neglected by previous studies in which a response within a particular time window was required (but see (Keil \& López-Moliner, 2012; Landwehr, Hecht, \& Both, 2014) for some exceptions in coincidence timing and motion extrapolation tasks respectively). Several monocular and binocular cues both retinal and extra-retinal contribute to the perception of speed in MID, which is difficult and prone to 
individual differences (Brenner, van den Berg, \& van Damme, 1996). Moreover, speed discrimination thresholds are usually higher for MID than for lateral motion (Aguado \& López-Moliner, 2019; Rushton \& Duke, 2009)). It has also been shown that differences in perceived speed depend on which part of the retina is stimulated (Brooks \& Mather, 2000; Murdison, Leclercq, Lefèvre, \& Blohm, 2019) and well known biases in the perceived spatial trajectories (Aguado \& López-Moliner, 2019; Harris \& Dean, 2003; Lages, 2006; Murdison et al., 2019; Rokers, Fulvio, Pillow, \& Cooper, 2018; Welchman, Tuck, \& Harris, 2004) or in motion extent in depth (Lages, 2006). The variability in the perception of MID, including speed and direction, makes it worth studying the performance of response timing when dealing with objects moving in depth. While most of the studies interested in MID have used perceptual judgements of speed or direction, timing performance has not been tested. An exception of interceptive timing tasks with motions other than linear or fronto-parallel are all the studies involving parabolic motion in depth (e.g. head-on approach trajectories), which have been motivated by testing specific models usually involving internalized variables like Gravity or physical size (Aguado \& López-Moliner, 2021; de la Malla \& López-Moliner, 2015b; Diaz, Cooper, \& Hayhoe, 2013; Diaz, Cooper, Rothkopf, \& Hayhoe, 2013; La Scaleia, Zago, \& Lacquaniti, 2015; Russo et al., 2017; Zago, McIntyre, Senot, \& Lacquaniti, 2008)

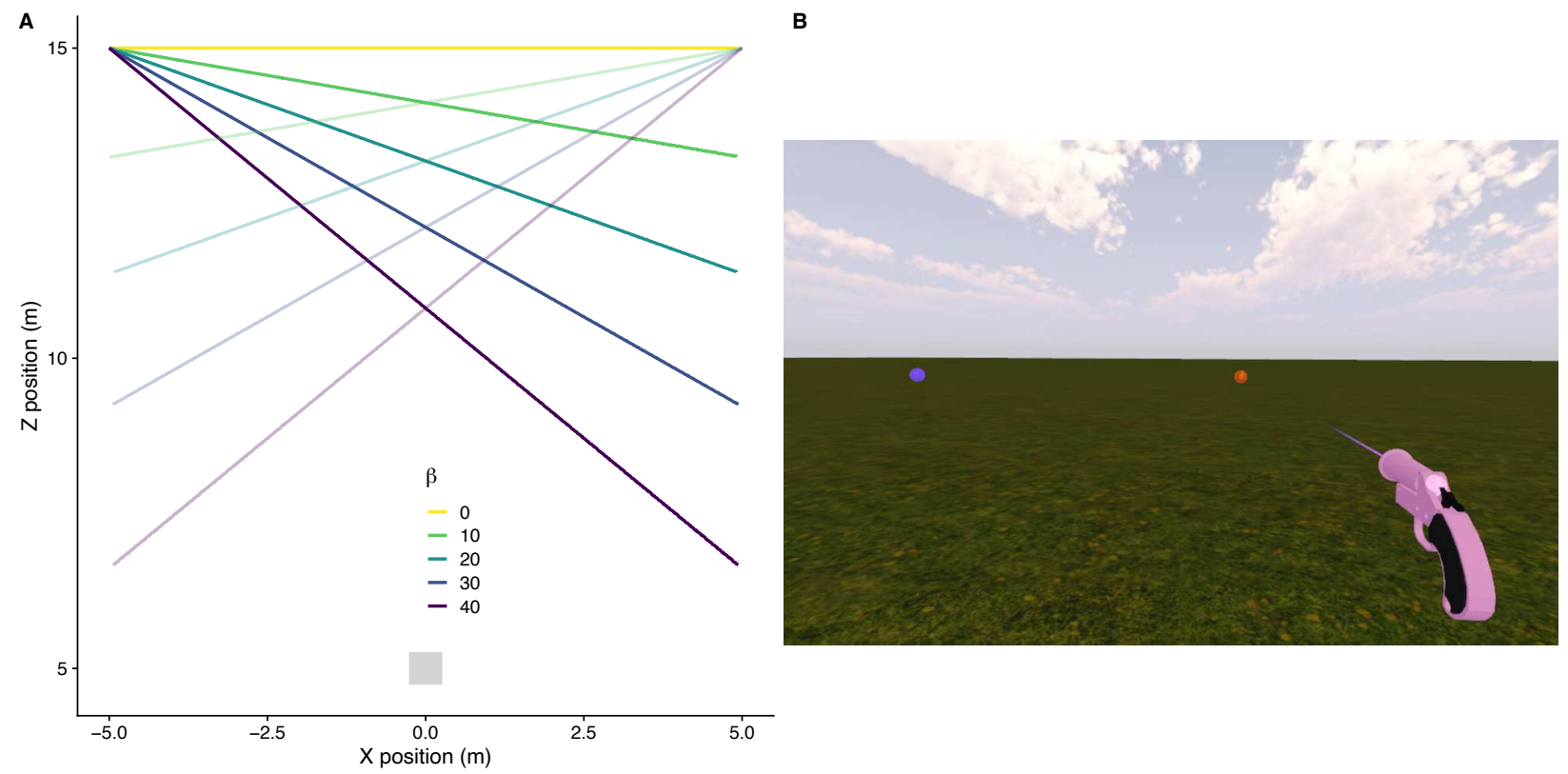


Figure 1. Trajectories in depth and illustration of the task. (A) Different possible trajectories described by the moving target. The starting position $(x, y)$ of the target could be $(-5,15)$ and then moved rightwards or $(5,15)$ and moved leftwards (trajectories with alpha transparency). The angle relative to the initial position of the participant $(0,5)$ could vary from 0 deg (yellow) to 40 deg (violet). (B) The moving target (red ball) approaches the interception or shooting location (violet) sphere; both target and shooting location have the same simulated physical size. The same grass texture was used in the two conditions (see Methods).

In this study we used immersive virtual reality (VR) to look at whether and if so, how, performance (defined as temporal errors) differs across multiple movement trajectories in a shooting task. Figure 1A shows a top-view of the different trajectories used in our Experiment: with one of them consisting of fronto-parallel motion (yellow trajectory) that corresponds with an approaching angle of $\beta$ $=0$ degs. Four other trajectories with different approaching angles $(\beta=10,20,30$ and 40 degs) were used. These different spatial paths were combined with three different speeds within an immersive Virtual Reality environment (see Figure 1B for an illustration of the 3D scenario) in which participants had to shoot at a moving target (red ball in Figure 1B) at an indicated shooting location (violet sphere in Figure 1B). Since we projected stereoscopic images, both monocular and binocular cues were available and we did not limit the number of these cues or introduce any conflict between them besides the conflicting use of accommodation(Wann, Rushton, \& Mon-Williams, 1995). It is known that the use of binocular cues (e.g. binocular disparity or change of disparity over time) to recover MID or the different approaching distances often depends on the level of experience of the participant with VR settings (Fulvio, Ji, Thompson, Rosenberg, \& Rokers, 2020) and that non-experienced users would rely more on monocular cues like expansion (see the projected size difference in Figure 1B). We did not constraint the tracking strategy (which was inferred from the measurements of head movements only) used by participants in any way, and so it was the one resulting of their own natural behavior or choices in exploring ways of solving the task. By using different tracking strategies people can exploit more foveal or peripheral information. 
This is important because some relevant cues for MID are perceived differently in the fovea than in the periphery. For example, speed of MID is underestimated in the periphery (Brooks \& Mather, 2000), suggesting a contribution of inter-ocular velocity differences in speed perception in depth (Fernandez \& Farell, 2005; Shioiri, Saisho, \& Yaguchi, 2000).

\section{Methods}

\section{Participants}

Fourteen subjects (age range 22-33, 7 males) participated in the experiments. Twelve of them were right-handed and two were left-handed as by self-report. All of them had normal or corrected to normal vision (with contact lenses), and none had evident motor abnormalities. All subjects gave written informed consent to take part on the study. The study was approved by the ethical committee of the University of Barcelona (IRB00003099) and followed the requirements of the Helsinki convention. All subjects gave written informed consent to take part on the study.

\section{Apparatus.}

The experiment was ran by an Intel i7-based PC (Intel, Santa Clara, CA). The stimuli were rendered by an NVIDIA GeForce GTX 1070 and sent for display to an HTC Vive Pro head-mounted display (HMD) at $90 \mathrm{~Hz}$ per eye $(2880 \mathrm{x} 1600)$. The field of view (FOV) of the HMD is 100 degs (horizontal) by 110 degs (vertical) for each eye. The head position in $3 \mathrm{D}$ coordinates (x, y and z) and the angular information (yaw, pitch and roll) were recorded by two SteamVR BASE STATION 2.0 at $90 \mathrm{~Hz}$. The response was registered through one of the headset controllers.

\section{Stimuli}

The virtual scene and stimuli were created with Unity software (Unity technologies, San Francisco, CA). Moving stimuli or targets consisted of a red sphere of $22 \mathrm{~cm}$ of diameter (same size as a soccer ball without familiarity cues) that appeared at one of two possible initial positions (see Figure 1A). 
After a period of $2.22 \mathrm{~s}$ the target started move at constant speed along a straight trajectory. The speed at which the target moved could be either $5.56,7.14$ or $10 \mathrm{~m} / \mathrm{s}$ and was randomized across trials. The direction of motion (leftwards or rightwards) was also varied on a trial-to-trial basis. Figure 1A shows all the possible trajectory angles $(\beta)$ between the target and the initial position of the observer (grey square at position $(0,5)$ in Figure 1A). The angle ( $\beta$ in Figure 1A) could be 0 (fronto-parallel motion), 10, 20, 30 or 40 degs.

The target moved at the designated speed towards the shooting location denoted by a violet sphere (same size of the target) that was located at a distance of $10 / \cos (\beta) \mathrm{m}$, from the initial position of the target (i.e. at $10 \mathrm{~m}$ when $\beta=0$ and at $13.05 \mathrm{~m}$ when $\beta=40$, see Figure1B). The target intersected the shooting location without stopping and continued to move until 1 second after the intersection. The time that it took for the target to reach the shooting location ranged between 1 and $2.34 \mathrm{~s}$ across all $\beta$ angles and targets' speeds.

\section{Procedure}

Each trial started when the participant entered a white square $(50 \times 50 \mathrm{~cm})$ rendered on the floor at position $(0,5)$ (shown in grey color in Figure 1A). Once the sensors detected that the HMD was within this area, a beep indicated that the trial was about to start. The square then disappeared, and both the target and designated shooting location appeared at the same time. After $2.22 \mathrm{~s}$ the target started move towards the designated shooting location and the task for the participant was to shoot at the target and try to hit it when the target was within the shooting location (violet sphere).

If the target was hit while overlapping with the shooting location, the violet sphere turned into a green circle. If the shot reached the designated shooting location (violet sphere) when the target was not there, it turned into a red circle. Pilot sessions showed that the task was quite difficult (less than $20 \%$ hits) in terms of getting positive feedback based on the overlapping of target and shooting location. We thus relaxed the temporal constraints to increase the proportion of trials in which positive feedback was 
provided in order to keep participants motivated. Therefore, we used twice the size of the target as a boundary to define the overlap with the shooting location and give feedback. Participants never reported being aware of this manipulation after questioning them at the end of the whole experiment.

\section{Conditions}

The study had two blocked conditions (named Delayed effect and Immediate effect) that were run in counterbalanced order across participants.

Delayed effect In this condition we examined performance in the shooting task when the effect of the action was delayed. The shooting task was implemented by triggering a bullet with a gun that was rendered at the same position of the hand-held controller. The bullet was represented by a pink sphere (diameter of $4.4 \mathrm{~cm}$ ) that travelled at $55 \mathrm{~m} / \mathrm{s}$. This speed will introduce a delay between the moment at which the participant pulls the trigger and the moment at which the bullet reaches the target when it is within the shooting location (as would happen when shooting with a real gun). The delay in reaching the designated shooting location when the trajectory had an angle $\beta=40$ degs was $0.111 \mathrm{~s}$ longer than when the $\beta=0$ deg. Participants were not explicitly told about the delays.

Immediate effect In this other condition, we replaced the bullet by a simulated instant laser transforming the task in a typical coincidence timing task (Aguilar-Lleyda et al., 2018; López-Moliner, Field, \& Wann, 2007; Tresilian, 1995). In that case, when participants pulled the trigger they briefly saw a pink line extending from the gun onwards. By using a laser, the differential delayed impact between the different trajectory angles disappeared. That means that the laser immediately intersected the target's path. This condition allows then to see whether any systematic error in the delayed effect condition is due to the differential time that it took for the bullet to intersect the different trajectories $(\beta)$ the target could follow.

In both conditions targets moved on a fine-grained grass textured background that did not provide any landmark or familiar size cue (see Figure 1B). Each condition consisted of four sessions of 180 trials 
( 5 angles $\times 3$ speeds $\times 2$ directions $\times 6$ repetitions) per session. That makes a total of 1440 trials (180 trials $\times 4$ sessions $\times 2$ conditions). The two conditions were blocked but the order of the type of condition was random across participants. Participants had a break between blocks for as long as they wanted. If they needed to rest during the session, they could also do so by not standing in the square that was projected on the floor at the beginning of each trial. In all cases, participants were told that they were free to move during the trial. Before starting the first session they were shown the spatial boundaries (space dimensions) and were aware that a grid appeared on the visual field if they were about to trespass the boundaries.

\section{Data analysis and hypothesis testing}

We registered the time at which participants pulled the trigger in both conditions and the temporal (and spatial) error in each trial. In the immediate effect condition, the time at which the trigger is pulled is the same as the time at which the laser crosses the target's path. In the delayed effect condition, the moment at which the trigger is pulled precedes the moment at which the bullet crosses the target's path. In addition to these variables, we also recorded the orientation and position of the head. Since we did not record eye movements we have to infer, and consequently be cautious, where participants might be looking by checking the head orientation in combination with the field of view of the HMD.

Since we instructed participants to hit the target in the shooting location defined by the violet sphere, we only had temporal errors associated to responses (López-Moliner, Vullings, Madelain, \& Beers, 2019), except for the trials in which subjects missed the shooting location (3.9\% of the trials). The temporal error between when the bullet reached the designated shooting location and when the target did so. The trials in which the designated shooting location was not hit $(3.9 \%)$ were excluded from the analysis. The temporal errors and the proportion of hits are the dependent variables we use to define performance. 
Tracking strategy We also analyze the head position and orientation during the target's motion and categorize every trial into one of two possible groups depending on the head movement. A trial was considered as tracking the trajectory if the change in the yaw angle was larger than 20 degs during the target motion. Otherwise, it was considered as tracking the end-point. The 20 degs threshold value was chosen after manually checking a sample of 100 trials across different participants and obtaining a 99\% accuracy in classifying the trials as following one or the other tracking strategy.

From previous literature in TTC, we know that people tend to commit later temporal errors for faster speeds for both lateral motion (Aguilar-Lleyda et al., 2018) and MID (Keil \& López-Moliner, 2012; López-Moliner et al., 2007). We expect then overall differences in performance depending on the target speed, e.g. later responses for faster targets. Also we will look at whether the differences in speed lead to equivalent differences in the temporal error for the different angles (e.g. different degrees of MID).

Concerning the tracking strategy, previous work has shown that both predictions about how targets will continue moving as well as interactions with moving targets are improved when one does pursue the target with one's gaze rather than when fixating somewhere else (de la Malla, Smeets, \& Brenner, 2017; Fooken \& Spering, 2019; Spering, Schütz, Braun, \& Gegenfurtner, 2011). Moreover, the speed of targets is better estimated when one directs the gaze at them than when fixating (Goettker, Braun, Schütz, \& Gegenfurtner, 2018; Goettker, Brenner, Gegenfurtner, \& Malla, 2019). For these reasons, we expect differences in the shooting performance depending on the tracking strategy, with smaller errors when tracking the trajectory than when tracking the end-point.

We will performed ANOVAs to compare differences between different conditions involving the approaching angle, the speed and the condition (delayed vs immediate effect). $\chi^{2}$ was be used to compare fractions of hits between different conditions. We will use Linear Mixed Models (LMM) to obtain estimation of parameters (e.g. slopes of temporal errors against angle $\beta$ ) and conduct the ANOVAs on the output of the lmm when the analysis involves the tracking strategy, since this is an ad hoc variable 
depending on individual strategies and will likely lead to an unbalanced design, which are better handled by LMM.
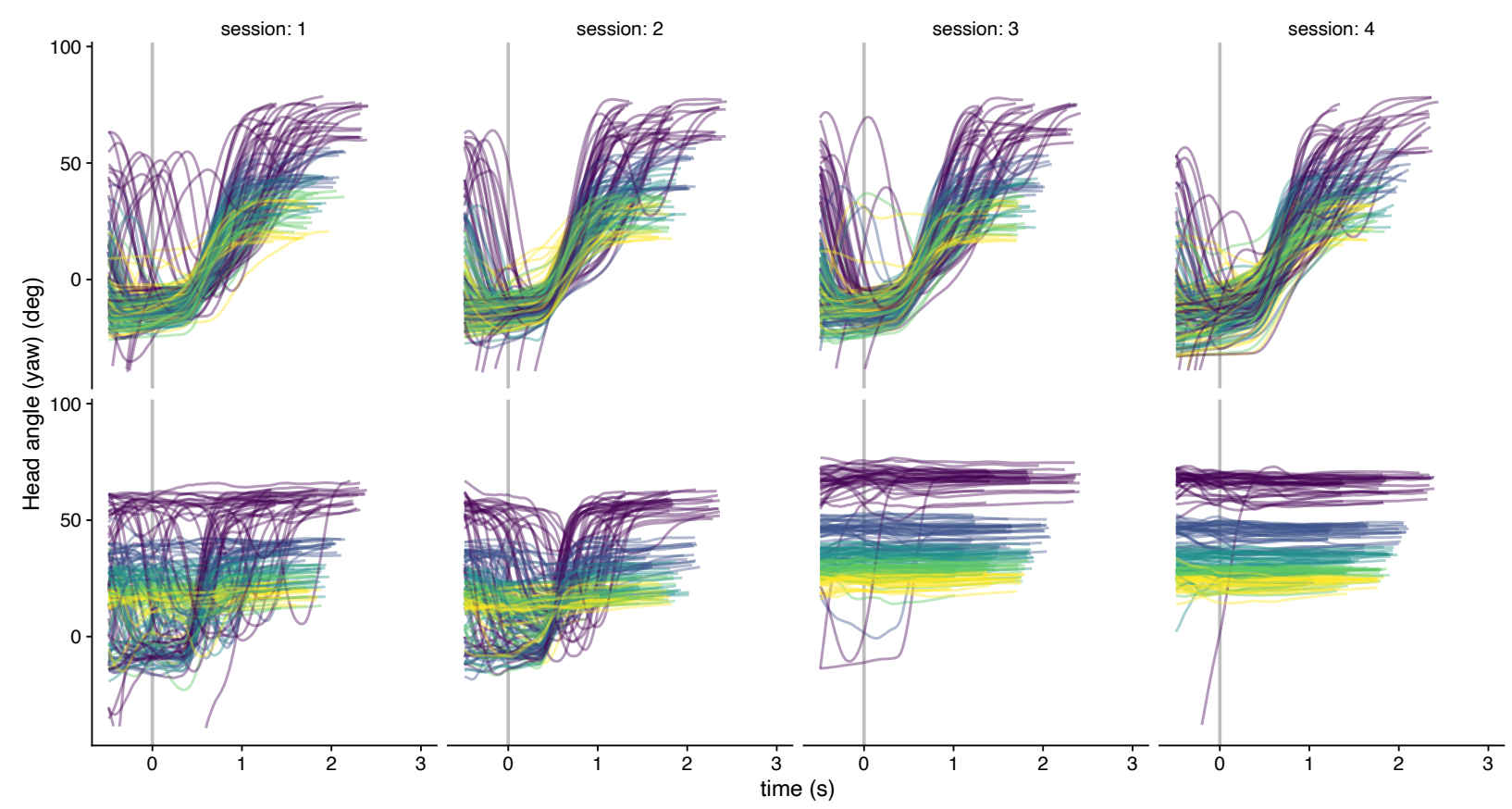

Figure 2. Examples of two participants (\#1 and \#5 in Figure 3; bottom and lower panels, respectively) as a function of time. The lines show the Head angle (yaw) for the individual trials of each session (different columns) of the delayed feedback condition. The yaw values have been normalized for the two directions. A yaw angle of 0 deg corresponds to the head oriented straight-ahead (e.g. looking at (0, 15) from (0, 5)). The vertical line denotes the stimulus motion onset. The participant in the upper panels always tracks the motion trajectory with the head, while the participant in the bottom panels initially interleaves two tracking strategies in sessions 1 and 2, and finally fixates on the end-point of the trajectory (end-point tracking strategy). The different colors code the approaching angle $\beta$ as in Figure $1 \mathrm{~A}$.

\section{Results}

Figure 2 shows the head orientation of two participants across time for all trials and sessions in the delayed effect condition. This figure illustrates the two main tracking strategies followed by the participants. The participant in the upper panels (participant \#1 in Figure 3) consistently tracks the target's 
trajectory with the head, while the participant in the bottom panels (participant \#5 in Figure 3) tracks both the trajectory and the end-point in the first two sessions and ends up with tracking the end-point in the two last sessions (constant yaw angle through all the target motion).

Figure 3 shows the proportion of times that each participant (in different panels) used the endpoint tracking strategy across sessions for the two conditions (color-coded). Participant \#1, for example, almost always tracks the trajectory (shown in the upper panels of Figure 2). This strategy is favoured by participants \#1, \#4 and \#11, as well as by \#3 in the delayed effect condition (black bars) and by \#8 in the immediate effect condition (yellow bars). Other participants (e.g. \#5 in the delayed effect condition, or \#12 and \#14 in both conditions) predominantly used the end-point tracking strategy.

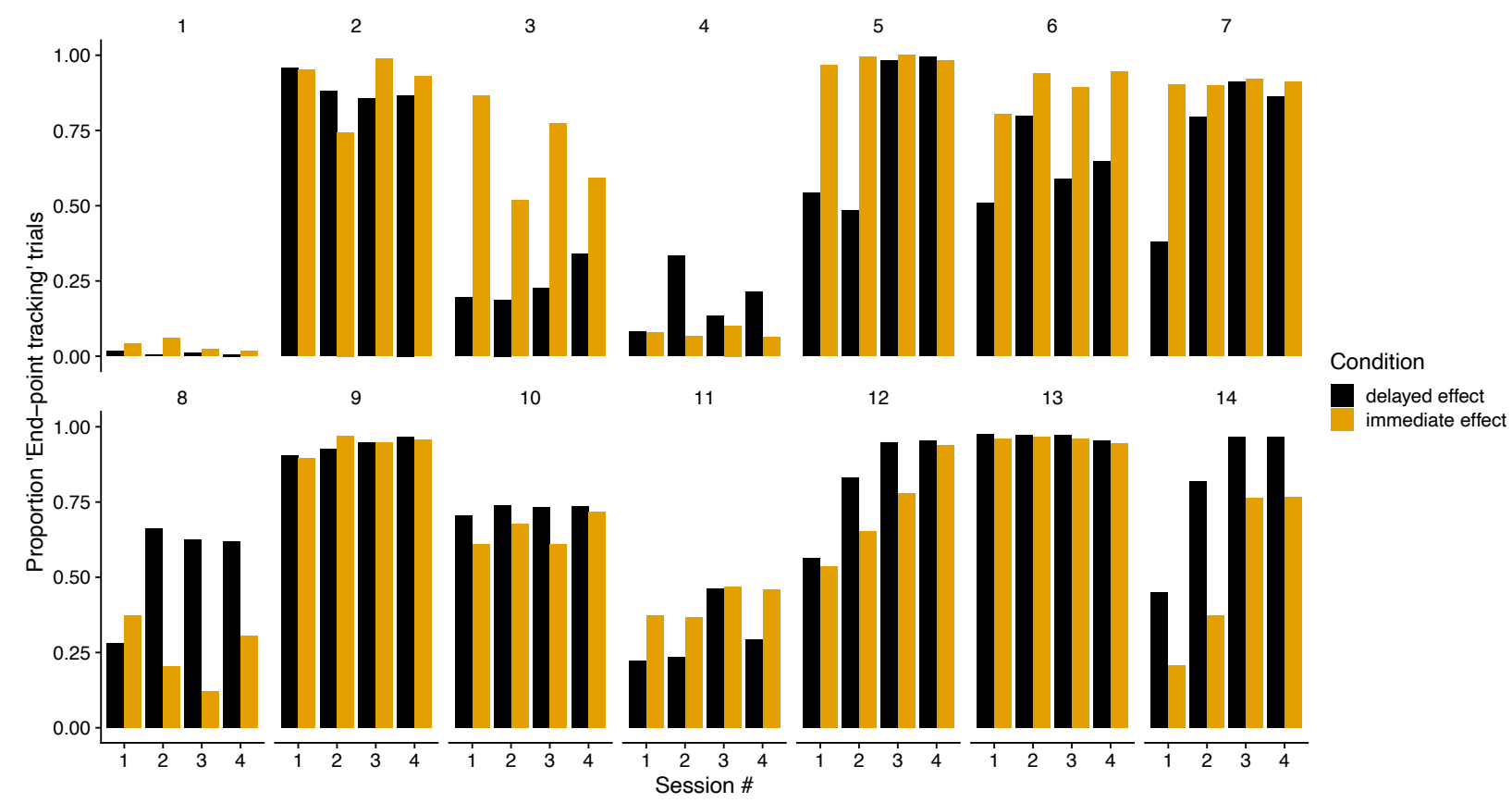

Figure 3. Proportion of trials in which participants adopted the end-point tracking strategy (i.e. the head was oriented towards the shooting location during all the trajectory). This proportion is shown for the two conditions (color coded) and for each participant (different panels) as a function of the session number. 
Performance: temporal error Figure 4 shows the temporal error for the two conditions as a function of the angle of approach $\beta$. Each participant is shown on a different panel. There is a very clear and consistent trend in the temporal error with $\beta$ : the error gets more positive (earlier responses) with larger values of the angle. This trend is much clearer in the delayed effect condition (i.e. the simulated bullet). The dashed lines denotes what would be the predicted slope of the temporal error if participants had not not taken into account the temporal differences of the bullet intersecting the target's paths for the different angles. Except for participants 6 and 8, the estimated slope of the temporal error is smaller than that predicted if the differential delay had not been considered. However, the immediate effect condition also shows a significant trend of the temporal error despite the laser intersecting the target's path at the same time as the trigger is pulled (and thus, with no delay for the different trajectory angles). The ANOVA on the temporal error produced a significant effect of condition $(F(1,13)=23.24, p<0.001$, $\left.\eta^{2}=0.185\right)$ and angle trajectory $\left(\mathrm{F}(1,13)=93.05, \mathrm{p}<0.0001, \eta^{2}=0.32\right)$. The interaction between condition and angle was significant $\left(\mathrm{F}(1,13)=57.22, \mathrm{p}<0.0001, \eta^{2}=0.10\right)$. This is consistent with the slope of the temporal error as a function of $\beta$ in the delayed effect condition being larger than the slope in the immediate effect condition. However, we want to know whether the slope of the temporal error in the immediate effect condition is significant, or in other words, whether $\beta$ significantly contributed to the temporal error. To test this, we compared two nested linear models in the immediate effect condition: a null model with only the intercept and a model with $\beta$. A likelihood ratio test $\left(\chi^{2}=36.563, \mathrm{df}=1, \mathrm{p}<0.001\right)$ reported a significant effect of the $\beta$ with a slope of $0.51 \mathrm{~ms}$ per unit of degree angle. 


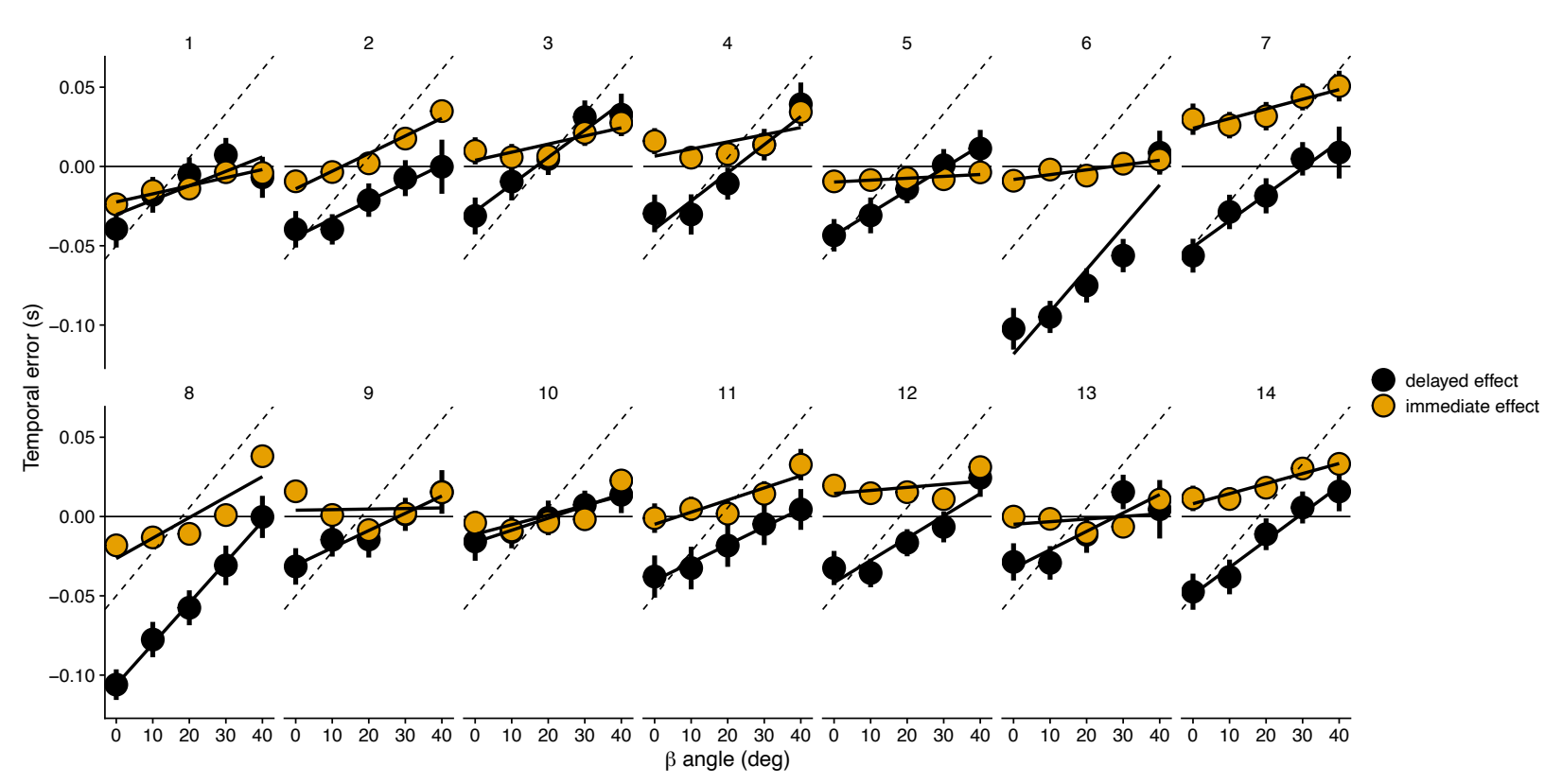

Figure 4. Temporal errors as a function of the $\beta$ angle for the two conditions (color coded). Each participant is plotted on different panel. The dashed line indicates the errors participants would made if the differential time it took for the bullet to reach intersect the target's path in the delayed effect condition had been ignored. Negative values denote late responses.

Effect of strategy on performance The effect of the angle trajectory on the observed bias in the temporal error is clear and very consistent across participants. The larger effect in the delayed effect condition could suggest that participants did not fully accounted for the differential time delay of the bullet in the different angles. It seems that some of the participants did not compensate at all since the slope appears to be very close to that predicted by the differential travel delay. However, the results in the immediate effect condition (in which there is no differential travel delay between angles) are also clear and suggest that the temporal bias is caused by the angle of approach rather than by whether the differential delays across trajectory angles are considered.

Figure 5A shows the mean temporal error per angle of approach and the two tracking strategies. Although there is a trend for earlier (more positive) responses when people tracked the trajectory, the 
strategy did not have a significant effect on the temporal error $(\mathrm{F}<1, \mathrm{p}=0.43)$. The interaction $\beta \times$ tracking strategy failed to reach significance too $(\mathrm{F}=2.61, \mathrm{p}=0.11)$.

A

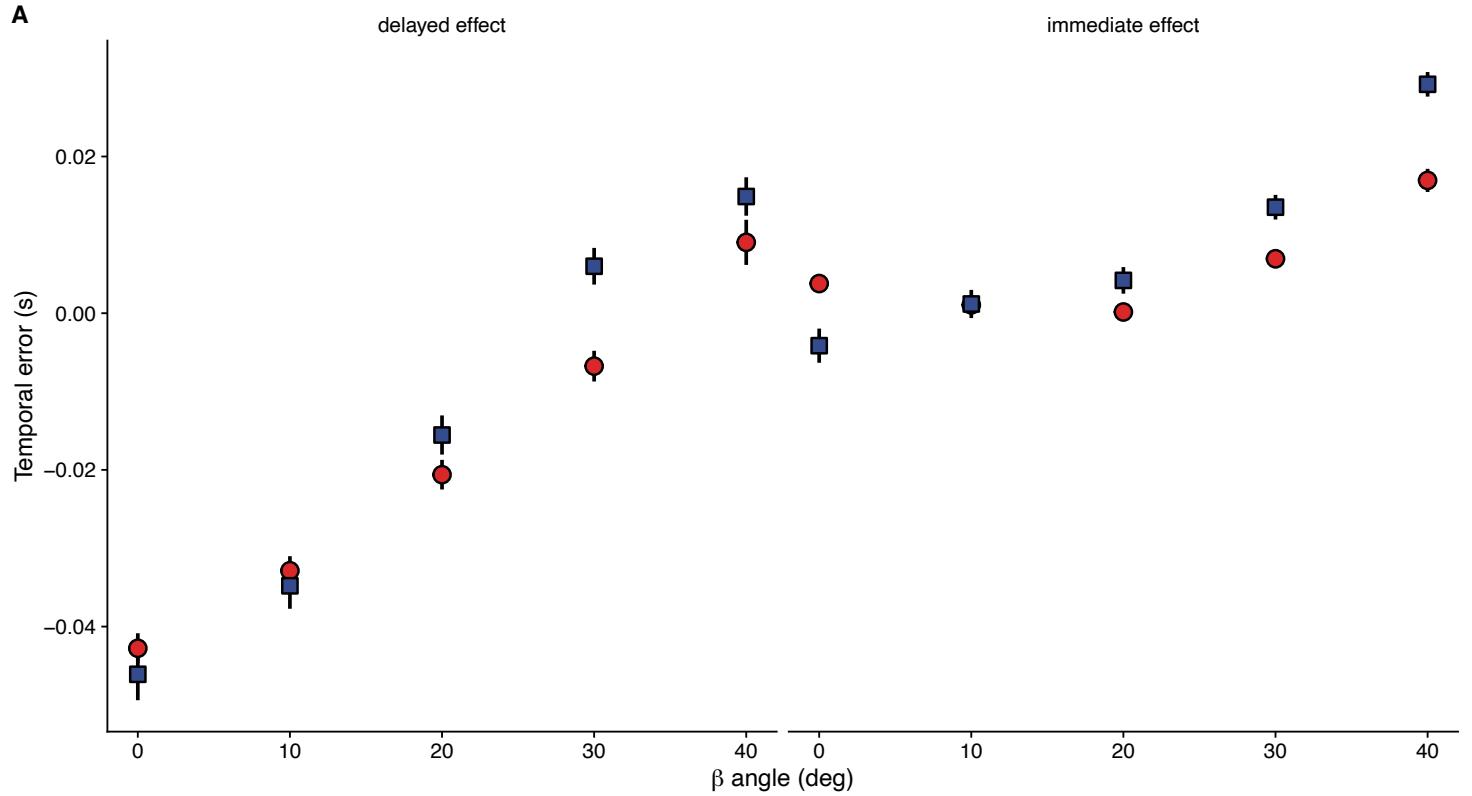

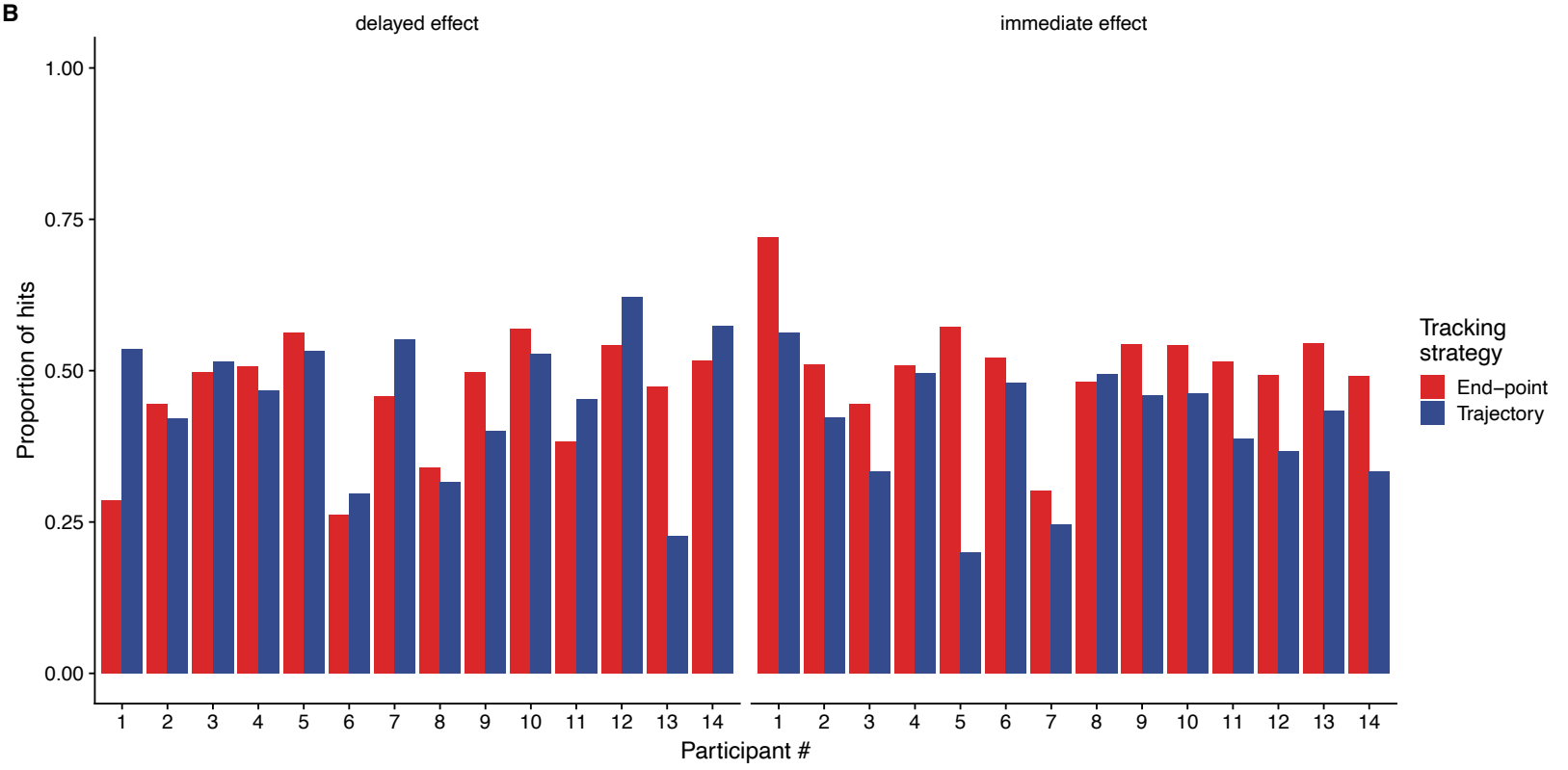

Figure 5. (A) Mean temporal error as a function of the angle of approach for the two conditions (in different panels) and split per tracking strategy (color and shape coded). Error bars denote 95\%-CI. (B) Proportion of hits (a hit was scored whenever the target was intercepted while overlapping with the 
sphere representing the interception zone) per participant in the two conditions (different panels) and split by the tracking strategy (color coded) in individual trials.

In terms of hits there was no significant difference between the two conditions (Figure 5B). The proportion of hits was $47 \%$ in the delayed effect conditions versus $48 \%$ in the immediate effect condition $\left(\chi^{2}(1)=1.6, p=0.2\right)$. We also looked at the effect of the tracking strategy within each condition. The tracking strategy did not make any difference in the delayed effect condition (47.3\% (end-point) versus $47.7 \%$ (trajectory), $\chi^{2}(1)=0.1, p=0.76$ ). However, in the immediate effect condition the end-point tracking strategy led to a larger proportion of hits than the trajectory tracking strategy $(50.1 \%$ versus $\left.45.3 \%, \chi^{2}(1)=19.4, p<0.001\right)$

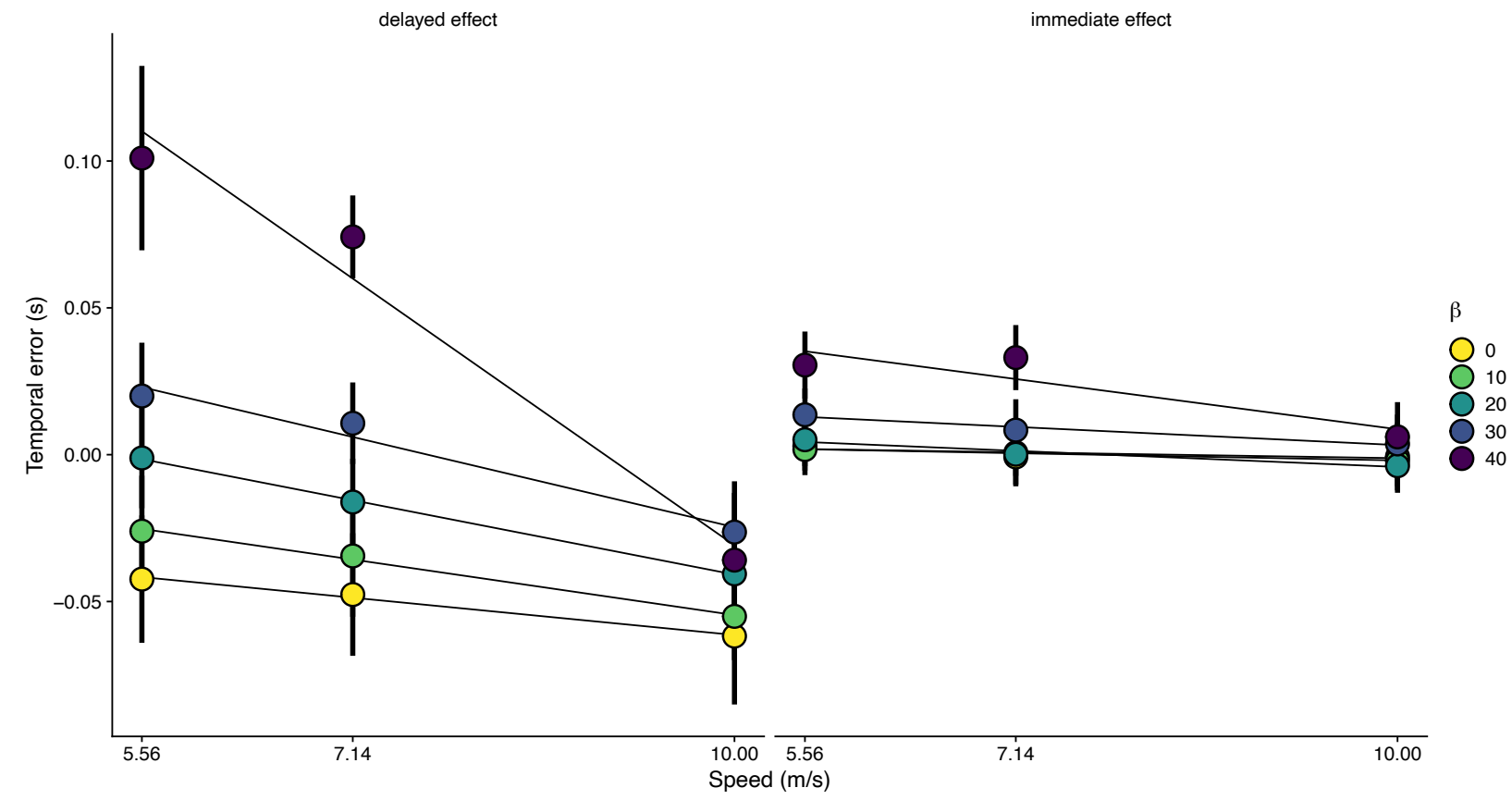

Figure 6. Temporal error as a function of target speed for the two conditions (different panels) and split per approaching angle of trajectory ( $\beta$, color-coded). Negative values denote later responses.

\section{Target speed analysis}

We used three different target speeds and wanted to know whether they affected the temporal error. Figure 6 shows the average temporal error against target speed for the different $\beta$ angles and the 
two conditions. By visual inspection one can see clear differences in the pattern of temporal errors between the two conditions.As expected, faster speeds provoke later temporal errors and the effect of speed on the temporal error is larger in MID (notice how the error changes as a a function of speed for the different values of the $\beta$ angle). We ran an ANOVA on the temporal error with speed, condition and $\beta$ as within subjects variables. Both condition and the $\beta$ angle had a significant effect on the temporal error as expected from the previous analysis (see above), but speed did not have a significant (main) effect $(\mathrm{F}(2,26)=0.34, \mathrm{p}=0.71)$ on the temporal error. Yet, the interaction between speed and angle of approach $(\beta)$ was significant $(\mathrm{F}(2,26)=31.7, \mathrm{p}<0.001)$ as well as the triple interaction speed $\times$ angle $\times$ condition $(\mathrm{F}(2,26)=13.28, \mathrm{p}<0.001)$. These results reflect the larger effect of speed as the $\beta$ angle gets larger (double interaction) and that this trend is much stronger in the delayed effect condition (triple interaction).

This larger effect of target speed on the temproal error in the delayed effect condition suggest that speed can be used differently in the two conditions. Since the final temporal error is basically determined at the moment of the response, it is worth looking at the position of the target when the response was triggered. In the immediate effect condition the moment of the response is the same as the moment the target is hit, or missed, since there is no travel time. 


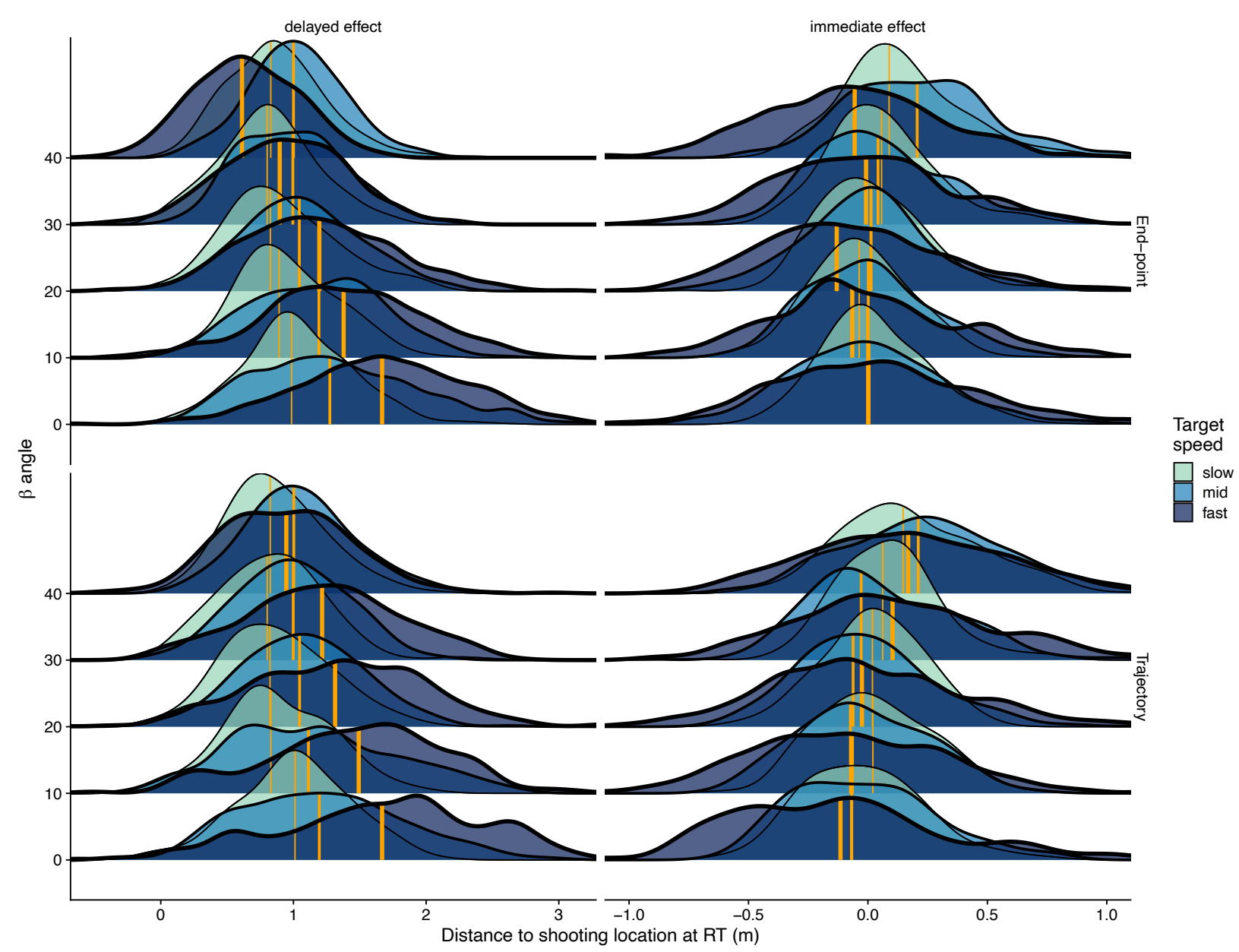

Figure 7. Distributions of distance between target and the shooting location at the moment of the response for the two conditions (column-wise panels) and tracking strategy (row-wise panels). Within each panel we show the densities for the different angles ( $\beta$ values) and target speeds (different fill color and line width). The vertical orange lines denote the medians of the corresponding distribution (the thicker the line the faster the speed).

Figure 7 shows the distributions of the distance between the target and the shooting location at the time the trigger was pulled (reaction time, RT). The distribution is shown separately for each angle, condition and speed. Since we are including the tracking strategy as a variable in addition to speed and $\beta$ angle, we conducted an ANOVA on the distance to the shooting location for each condition separately in order to make the interpretation simpler. The ANOVA was run on a LMM (see methods). The model 
included $\beta$ angle, target speed and tracking strategy as fixed effects and subjects was considered as random effect.

In the delayed effect condition, the ANOVA yielded very significant main effects of approaching angle $(\beta)(\mathrm{F}(1,366)=124.4, \mathrm{p}<0.001)$, target speed $(\mathrm{F}(1,366)=77.4, \mathrm{p}<0.001)$ and the interactions $\beta \times$ tracking strategy $(\mathrm{F}(1,366)=9.2, \mathrm{p}=0.0026)$ and $\beta \times \operatorname{speed}(\mathrm{F}(2,366)=36.2, \mathrm{p}<0.001)$. No other effect was significant. In the immediate effect condition similar significant effects were obtained except for the effect of target speed which was marginal $(\mathrm{F}(2,357)=2.85, \mathrm{p}=0.06)$. The angle of approach was significant $(\mathrm{F}(1,358)=38, \mathrm{p}<0.001)$ as well as the interactions $\beta \times$ tracking strategy $(\mathrm{F}(1,357)=10.12, \mathrm{p}=0.0015)$ and $\beta \times$ speed $(\mathrm{F}(2,357)=6.1, \mathrm{p}=0.0025)$. The effect of the tracking strategy then was not significant in either condition but it interacted significantly with $\beta$ in both of them. Next, we will elaborate on these different effects.

In the delayed effect condition, people pulled the trigger when the distance between the target and the shooting location was larger for faster speeds. This makes sense because faster targets will travel larger distances than slower ones for a similar arrival time to the shooting location. Yet, this anticipation was not enough to compensate for speed differences and made participants be $23 \mathrm{~ms}$ late in the faster speed relative to the slower one $(t=8.59, \mathrm{p}<0.001)$. However, the difference of target-shooting location distances at the response time between speeds gets smaller as the MID increases (i.e. for larger $\beta$ ) as can be noted by the separation between the medians of the distributions for the different speeds (orange vertical lines). In addition, when targets moved in depth (larger angles) people responded (i.e. pulled the trigger) when the distance between the target and the shooting location was smaller. This is consistent with compensating for the travel delay of the bullet. Interestingly, however, the rate at which the distance was shortened with $\beta$ in the delayed effect condition, was different depending on the tracking strategy. When participants tracked the end-point, as $\beta$ increased, they responded when the target got progressively closer at a rate of $1.18 \mathrm{~cm} / \mathrm{deg}(0.56,0.7$ and $2.29 \mathrm{~cm} / \mathrm{deg}$ for the slow, mid and fast speed respectively). This rate (i.e. the slope of the distance to the shooting location against $\beta$ ) was smaller $(0.3 \mathrm{~cm} / \mathrm{deg})$ when 
participants tracked the trajectory $(0.1,0.6$ and $0.2 \mathrm{~cm} / \mathrm{deg}$ for the slow, mid and fast speed respectively) consistent with the significant interaction $\beta \times$ tracking strategy reported above. This different pattern between the two strategies can be explained by participants perceiving MID differently in the fovea and in the periphery. More concretely, the shorter distance of the target to the shooting location when they tracked the end-point and MID was present is consistent with underestimating the speed in the periphery (Brooks \& Mather, 2000).

A slightly different pattern is observed in the immediate effect condition. Figure 7 shows how the distance between the target and the shooting location is more similar across speeds (the main effect of speed was marginal). Since the response time is the same as the interception time in this condition, this similarity is, in part, trivial. However, the rate of change of the distance with the $\beta$ angle and the effect of the tracking strategy is also different. Unlike in the delayed effect condition, the average distance of the target to the shooting location increased with $\beta$. Note the progressive rightward shift of the vertical lines (i.e. of the medians of the distributions) in the immediate effect condition. When tracking the end-point, the distance to the shooting location increased at an average rate of $0.17 \mathrm{~cm} / \mathrm{deg}(0.2,0.4,-0.09 \mathrm{~cm} / \mathrm{deg}$ for the slow, mid and fast speed respectively). Like in the delayed effect condition, the distance increased with $\beta$ at a larger rate $(0.28 \mathrm{~cm} / \mathrm{deg})$ when participants tracked the trajectory consistently with the significant interaction between $\beta$ and tracking strategy reported above for the immediate effect condition. This is again consistent with participants perceiving slower the speed in depth in the periphery. The difference, however, is smaller (end-point strategy: $0.17 \mathrm{~cm} / \mathrm{deg}$ vs trajectory strategy: $0.28 \mathrm{~cm} / \mathrm{deg}$ ) than in the delayed effect condition. The fact that we obtain smaller changes of distance between the target and the shooting location with $\beta$ in the immediate effect condition can be explained by the fact that the approaching angle $\beta$ does not introduce any travel delay. However, the differences between tracking strategies cannot be explained by these delays.

Position variability. 
In addition to the mean distances between the target and the shooting location at the moment at which the trigger is pulled, Figure 7 also shows a different pattern regarding positional variability between the two conditions. One can notice how in the delayed effect condition the width of the distributions seems to narrow as $\beta$ increases. This effect of the angle of approach is not clearly noticeable in the immediate effect condition; the width of the distributions does not seem to change with $\beta$. After computing the SD of the individual distributions across conditions, angle of approach and target speed, we conducted an ANOVA on the SD of distance distribution with these variables as independent variables. The main effect of $\beta$ was significant in the delayed effect condition $(\mathrm{F}(1,13)=41.45, \mathrm{p}<0.001)$ but not in the immediate effect condition $(\mathrm{F}(1,13)=0.002, \mathrm{p}=0.967)$. The average distance variability did not change with the $\beta$ angle when the effect was immediate (slope not significantly different from zero: slope $\left.=-8.836 \times 10^{-06}, \mathrm{t}=-0.020, \mathrm{p}=0.984\right)$, but it did significantly in the delayed effect condition (slope $=-0.243 \mathrm{~cm} / \mathrm{deg}, \mathrm{t}=-3.944, \mathrm{p}<0.001$ ). The positional SD then decreased at a rate of $0.243 \mathrm{~cm} / \mathrm{deg}$. Target speed had a very significant effect in both conditions $(F(1,13)=97.61, p<0.001$ and $F(1,13)=120.3$, $\mathrm{p}<0.001$ for the delayed and immediate effect conditions respectively). This is expected and well known in the interception literature as increasing speed makes positional judgements more difficult (Brenner, van Beers, Rotman, \& Smeets, 2006).

\section{Discussion}

In the present study we have shown that MID has an effect on the temporal errors people make in an interceptive timing task (shooting task in our case) that is very consistent across participants and conditions. When compared with fronto-parallel motion, performance when MID is present shows earlier final temporal errors. This conclusion is based on the fact that by increasing the angle of approach $(\beta)$ leads to more anticipatory responses. This pattern is present in the two studied conditions: delayed and immediate effects. Although part of this trend can be accounted for by the travel delay of the simulated bullet (i.e. the target gets closer to the observer as the angle $\beta$ increases), in the immediate effect condition 
there is no such delay and the trend is very significant and consistent across participants too. Apparently participants failed to fully recover the 3D speed when MID was present, in a similar way as failure of velocity constancy has been shown (McKee \& Welch, 1989) when there is a lack of familiar-size cues that could have otherwise helped scale the changing distance across the different angles, specially in VR settings (Distler, Gegenfurtner, Van Veen, \& Hawken, 2000). This effect in an interceptive timing task is consistent with more anticipatory errors for trajectories approaching the observer (MID) than for frontoparallel ones in a motion prediction task reported previously(Landwehr et al., 2014).

Accurate perception of MID (both speed and direction) accurately is a complex process that requires integrating extra-retinal (e.g. gaze direction) and retinal information so that $2 \mathrm{D}$ information from the retinae are properly mapping to a 3D layout but this transformation can be incomplete (Murdison et al., 2019). It is clear that the differences in temporal errors as a function of speed depended on the angle of approach, as shown in Figure 6. Another factor that seems to contribute to this incomplete transformation is the tracking strategy. Although we found no significant differences of the tracking strategy on the final temporal error, it did affect the response time through its interaction with the angle of approach, that is, when a MID component became more prominent (i.e. larger $\beta$ ).

People that did not track the trajectory with the head (end-point tracking) responded later, which would be consistent with them perceiving the speed in depth more slowly (Figure 5A). It is known that MID is perceived more slowly in the periphery (Brooks \& Mather, 2000) and part of the motion trajectory could have been perceived peripherally when people tracked the end-point. However, this pattern of late responses when the head was oriented towards the shooting area during all the trial (end-point tracking) did not undermine performance, and, on the contrary, even increased the proportion of hits in the immediate effect condition significantly. Previous research has shown that when participants are required to intercept targets at specific locations, they often rather look at the designated location during the trial or initially look at the target and then direct the gaze towards the indicated location (de la Malla, Rushton, Clark, Smeets, \& Brenner, 2019; de la Malla et al., 2017). The reasons why directing one's gaze towards 
a designated interception location are diverse. One is related to the possible size difference between the target and the interceptive location. If the interceptive location is smaller than the target, it requires a higher spatial precision and thus gaze is directed there (Brenner \& Smeets, 2015) probably to enhance the spatial resolution at that area. In our experiment, both the target and the designated shooting location had the same size, to not favor any of the strategies. By tracking the end-point people increase the spatial resolution by foveating the target around the moment of interest (Schütz, Braun, \& Gegenfurtner, 2009) and reduce the effects that saccades may have in judging how targets move that can give rise to certain perceptual errors (Goettker et al., 2018, 2019; Matziridi, Brenner, \& Smeets, 2015; Morrone, Burr, \& Vaina, 1995; Schlag \& Schlag-Rey, 2002) that could lead to errors in interception (de la Malla, Brenner, Haan, \& Smeets, 2019; de la Malla et al., 2018).

On the contrary, participants that tracked the trajectory with the head made more anticipatory responses in the both condition. It is known that keeping the eyes on the moving target enhances the prediction of visual motion (Spering et al., 2011), probably due to additional extra-retinal signals contributing to speed estimation. Anticipatory interception errors have been previously reported in similar interception tasks (Brenner, Cañal-Bruland, \& Beers, 2013; de la Malla et al., 2012, 2014, 2017). Apparently, both when one is allowed to intercept a target anywhere along its path or even when having to do so at an indicated location there is a tendency to hit ahead of the target (errors of $\sim 100 \mathrm{~ms}$ that become smaller as the precision required to successfully solve the task increases).

The two conditions regarding the type of effect leave us with some differences that need further discussion. One difference is that, independently of the tracking strategy, as MID increased the distance between the target and the shooting location at RT was shorter for faster speeds in the delayed effect condition than in the immediate effect one. The earlier responses for faster speeds when motion was fronto-parallel progressively became late responses with MID. This pattern does not bode well with participants only misjudging the direction in depth (Aguado \& López-Moliner, 2019; Harris \& Dean, 2003; Lages, 2006; Rokers et al., 2018; Welchman et al., 2004), that is underestimating $\beta$, because the 
target position would have been perceived more advanced than it actually was, leading to earlier responses. However, not only is speed in depth underestimated with respect to lateral movement (Brenner, Van Den Berg, \& Van Damme, 1997; Brooks \& Stone, 2006; Rushton \& Duke, 2009; Welchman, Lam, \& Bulthoff, 2008), but its discrimination thresholds are also known to be higher relative to fronto-parallel speed (Aguado \& López-Moliner, 2019) which makes more difficult to discriminate the different speeds for larger angles of approach. This explanation would be consistent with the fact that position variability becomes increasingly similar across speeds when MID is more present in the delayed effect condition. Why is this pattern is less clear in the immediate effect condition? In this task, the rate of change of the optical gap between target and interception zone becomes an additional relevant variable (Bootsma \& Oudejans, 1993; Landwehr et al., 2014) that is less affected by possible incomplete mappings from 2D retinal information to MID and could have been exploited by the participants.

Another difference between the two conditions is the absence of a significant difference between speeds in the immediate effect condition contrasting with the large effect of target speed in the delayed effect condition. One possibility is that velocity and position are used differently in these two conditions. Some studies have proposed that response initiation in interceptive timing tasks would be controlled by positional information (Aguilar-Lleyda et al., 2018; Brenner et al., 2006; Wann, 1996) whose state can be updated by velocity in a Kalman filter-like way (Aguilar-Lleyda et al., 2018). It could then be that response is mainly spatially controlled in the delayed effect condition, while velocity would have to be used as well in the immediate effect condition since it becomes a task-relevant variable at the very same moment of the response. This is so because the target speed constraints more clearly the temporal window in which a response has to be executed. Although this interpretation is speculative, there is previous evidence from the TTC literature that is consistent with it. When a coincidence timing task using a single key press was used to estimate the TTC of looming objects participants heavily weighted rate of expansion, an optical variable which contains spatio-temporal structure (López-Moliner et al., 2007). However, in a different study (López-Moliner \& Keil, 2012), where the interceptive action did not consist 
of a key press and movement time could be modulated, participants relied on visual angle and much less on rate of expansion.

\section{Conclusion}

We have reported that MID affects the temporal performance in an interceptive task: the angle of approach of a target with respect to an observer affects the moment of action response in systematic ways. The temporal requirements of the task defined by the type of action effect (delayed or immediate) need to be considered regarding target speed and the amount of MID present in the target trajectory.

\section{Acknowledgements}

This work was funded by the Catalan government (2017SGR-48) and grant ref. grant ref. PID2020-114713GB-I00 (AEI/FEDER, UE) and PID2020-116400GA-I00 (AEI/FEDER, UE). 


\section{References}

Aguado, B., \& López-Moliner, J. (2019). Perceived speed of motion in depth modulates misjudgements of approaching trajectories consistently with a slow prior. Vision Research, 159, 1-9.

Aguado, B., \& López-Moliner, J. (2021). Flexible viewing time when estimating time-tocontact in 3D parabolic trajectories. J Vis, 21(4), 9.

Aguilar-Lleyda, D., Tubau, E., \& López-Moliner, J. (2018). An object-tracking model that combines position and speed explains spatial and temporal responses in a timing task. Journal of Vision, 18(12), 1-19.

Bootsma, R., \& Oudejans, R. (1993). Visual information about time-to-collision between two objects. Journal of Experimental Psychology: Human Perception \& Performance, 19, 1041-1052.

Brenner, E., Cañal-Bruland, R., \& Beers, R. J. van. (2013). How the required precision influences the way we intercept a moving object. Experimental Brain Research, 230(2), 207-218.

Brenner, E., \& Smeets, J. B. J. (2009). Sources of variability in interceptive movements. Exp Brain Res, 195(1), 117-133.

Brenner, E., \& Smeets, J. B. J. (2015). How people achieve their amazing temporal precision in interception. J Vis, 15(3).

Brenner, E., van Beers, R. J., Rotman, G., \& Smeets, J. B. J. (2006). The role of uncertainty in the systematic spatial mislocalization of moving objects. Journal of Experimental Psychology: Human Perception and Performance, 32, 811-825.

Brenner, E., van den Berg, A. V., \& van Damme, W. J. (1996). Perceived motion in depth. Vision Research, 36, 699-706.

Brenner, E., Van Den Berg, A., \& Van Damme, W. (1997). Perceived motion in depth. Ophthalmic Literature, 1(50), 50.

Brooks, K., \& Mather, G. (2000). Perceived speed of motion in depth is reduced in the periphery. Vision Res, 40(25), 3507-3516.

Brooks, K. R., \& Stone, L. S. (2006). Stereomotion suppression and the perception of speed: Accuracy and precision as a function of 3D trajectory. Journal of Vision, 6(11), 6-6.

Brouwer, A.-M., Smeets, J. B. J., \& Brenner, E. (2005). Hitting moving targets: Effects of target speed and dimensions on movement time. Exp Brain Res, 165(1), 28-36. 
Cámara, C., López-Moliner, J., Brenner, E., \& Malla, C. de la. (2020). Looking away from a moving target does not disrupt the way in which the movement toward the target is guided. Journal of Vision, 20(5), 5-5.

de la Malla, C., Brenner, E., Haan, E. H. de, \& Smeets, J. B. (2019). A visual illusion that influences perception and action through the dorsal pathway. Communications biology, 2(1), 1-7.

de la Malla, C., \& López-Moliner, J. (2015a). Hitting moving targets with a continuously changing temporal window. Exp Brain Res, 233, 2507-2515.

de la Malla, C., \& López-Moliner, J. (2015b). Predictive plus online visual information optimizes temporal precision in interception. J Exp Psychol Hum Percept Perform, 41(5), 1271-1280.

de la Malla, C., López-Moliner, J., \& Brenner, E. (2012). Seeing the last part of a hitting movement is enough to adapt to a temporal delay. Journal of Vision, 12(10), 1-15.

de la Malla, C., López-Moliner, J., \& Brenner, E. (2014). Dealing with delays does not transfer across sensorimotor tasks. J Vis, 14(12).

de la Malla, C., Rushton, S. K., Clark, K., Smeets, J. B., \& Brenner, E. (2019). The predictability of a target's motion influences gaze, head, and hand movements when trying to intercept it. Journal of neurophysiology, 121(6), 2416-2427.

de la Malla, C., Smeets, J. B., \& Brenner, E. (2017). Potential systematic interception errors are avoided when tracking the target with one's eyes. Scientific Reports, 7.

de la Malla, C., Smeets, J. B., \& Brenner, E. (2018). Errors in interception can be predicted from errors in perception. Cortex, 98, 49-59.

Diaz, G., Cooper, J., \& Hayhoe, M. (2013). Memory and prediction in natural gaze control. Philos Trans R Soc Lond B Biol Sci, 368(1628), 20130064.

Diaz, G., Cooper, J., Rothkopf, C., \& Hayhoe, M. (2013). Saccades to future ball location reveal memory-based prediction in a virtual-reality interception task. Journal of Vision, 13(1):20, 1-14.

Distler, H. K., Gegenfurtner, K. R., Van Veen, H. A., \& Hawken, M. J. (2000). Velocity constancy in a virtual reality environment. Perception, 29(12), 1423-1435.

Fernandez, J. M., \& Farell, B. (2005). Seeing motion in depth using inter-ocular velocity differences. Vision research, 45(21), 2786-2798.

Fooken, J., \& Spering, M. (2019). Decoding go/no-go decisions from eye movements. Journal of vision, 19(2), 5-5.

Fulvio, J. M., Ji, M., Thompson, L., Rosenberg, A., \& Rokers, B. (2020). Cue-dependent effects of VR experience on motion-in-depth sensitivity. PloS one, 15(3), e0229929. 
Goettker, A., Braun, D. I., Schütz, A. C., \& Gegenfurtner, K. R. (2018). Execution of saccadic eye movements affects speed perception. Proceedings of the National Academy of Sciences, 115(9), 2240-2245.

Goettker, A., Brenner, E., Gegenfurtner, K. R., \& Malla, C. de la. (2019). Corrective saccades influence velocity judgments and interception. Scientific reports, 9(1), 1-12.

Harris, J. M., \& Dean, P. J. A. (2003). Accuracy and precision of binocular 3-d motion perception. Journal of Experimental Psychology: Human Perception and Performance, $5,869-881$.

Joerges, B., \& López-Moliner, J. (2019). Earth-gravity congruent motion facilitates ocular control for pursuit of parabolic trajectories. Scientific Reports, 9(14094), 1-13.

Keil, M. S., \& López-Moliner, J. (2012). Unifying time to contact estimation and collision avoidance across species. PLoS Comput Biol, 8(8), e1002625.

Kreyenmeier, P., Fooken, J., \& Spering, M. (2017). Context effects on smooth pursuit and manual interception of a disappearing target. Journal of neurophysiology, 118(1), 404415.

Kwon, O.-S., Tadin, D., \& Knill, D. C. (2015). Unifying account of visual motion and position perception. Proc Natl Acad Sci, 112(26), 8142-8147.

La Scaleia, B., Zago, M., \& Lacquaniti, F. (2015). Hand interception of occluded motion in humans: A test of model-based vs. On-line control. Journal of Neurophysiology, 114(3), 1577-1592.

Lages, M. (2006). Bayesian models of binocular 3-d motion perception. Journal of Vision, 6(4), 14-14.

Landwehr, K., Hecht, H., \& Both, B. (2014). Allocentric time-to-contact and the devastating effect of perspective. Vision Res, 105C, 53-60.

López-Moliner, J., Field, D. T., \& Wann, J. P. (2007). Interceptive timing: Prior knowledge matters. Journal of Vision, 7(13), 11, 1-8.

López-Moliner, J., \& Keil, M. (2012). People favour imperfect catching by assuming a stable world. PLoS ONE, 7(4), 1-8.

López-Moliner, J., Vullings, C., Madelain, L., \& Beers, R. J. van. (2019). Prediction and final temporal errors are used for trial-to-trial motor corrections. Scientific Reports, 9(1), 19230.

Matziridi, M., Brenner, E., \& Smeets, J. B. (2015). The role of temporal information in perisaccadic mislocalization. PloS one, 10(9), e0134081.

McKee, S. P., \& Welch, L. (1989). Is there a constancy for velocity? Vision Research, 29, 553561. 
Morrone, M. C., Burr, D. C., \& Vaina, L. M. (1995). Two stages of visual processing for radial and circular motion. Nature, 376, 507-509.

Murdison, T. S., Leclercq, G., Lefèvre, P., \& Blohm, G. (2019). Misperception of motion in depth originates from an incomplete transformation of retinal signals. Journal of vision, 19(12), 21-21.

Rokers, B., Fulvio, J. M., Pillow, J. W., \& Cooper, E. A. (2018). Systematic misperceptions of 3$\mathrm{d}$ motion explained by bayesian inference. Journal of vision, 18(3), 23-23.

Rushton, S., \& Duke, P. (2009). Observers cannot accurately estimate the speed of an approaching object in flight. Vision Res, 49, 1919-1928.

Russo, M., Cesqui, B., La Scaleia, B., Ceccarelli, F., Maselli, A., Moscatelli, A., Zago, M., et al. (2017). Intercepting virtual balls approaching under different gravity conditions: Evidence for spatial prediction. Journal of Neurophysiology, 118(4), 2421-2434.

Schlag, J., \& Schlag-Rey, M. (2002). Through the eye, slowly: Delays and localization errors in the visual system. Nature Reviews Neuroscience, 3, 191-200.

Schütz, A. C., Braun, D. I., \& Gegenfurtner, K. R. (2009). Object recognition during foveating eye movements. Vision research, 49(18), 2241-2253.

Shioiri, S., Saisho, H., \& Yaguchi, H. (2000). Motion in depth based on inter-ocular velocity differences. Vision research, 40(19), 2565-2572.

Spering, M., Schütz, A. C., Braun, D. I., \& Gegenfurtner, K. R. (2011). Keep your eyes on the ball: Smooth pursuit eye movements enhance prediction of visual motion. Journal of Neurophysiology, 105(4), 1756-1767.

Tresilian, J. R. (1995). Perceptual and cognitive processes in time-to-contact estimation: Analysis of prediction-motion and relative judgement tasks. Perception \& Psychophysics, 57, 231-245.

Wann, J. P. (1996). Anticipating arrival: Is the tau-margin a specious theory? Journal of Experimental Psychology: Human Perception and Performance, 22, 1031-1048.

Wann, J. P., Rushton, S., \& Mon-Williams, M. (1995). Natural problems for stereoscopic depth perception in virtual environments. Vision research, 35(19), 2731-2736.

Welchman, A. E., Lam, J. M., \& Bulthoff, H. H. (2008). Bayesian motion estimation accounts for a surprising bias in 3D vision. Proc Natl Acad Sci U S A, 105(33), 12087-12092.

Welchman, A. E., Tuck, V. L., \& Harris, J. M. (2004). Human observers are biased in judging the angular approach of a projectile. Vision Research, 44, 2027-2042.

Zago, M., McIntyre, J., Senot, P., \& Lacquaniti, F. (2008). Internal models and prediction of visual gravitational motion. Vision Res, 48(14), 1532-1538. 